\title{
Nasalance and nasality at experimental velopharyngeal openings in palatal prosthesis: a case study
}

\author{
Aveliny Mantovan LIMA-GREGIO', Viviane Cristina de Castro MARINO², Maria Inês PEGORARO-KROOK ${ }^{3}$, Plinio \\ Almeida BARBOSA ${ }^{4}$, Homero Carneiro AFERRI ${ }^{5}$, Jeniffer de Cassia Rillo DUTKA ${ }^{6}$
}

\author{
1- PhD, Researcher, Speech Prosody Studies Group, Institute of Language Studies (IEL), Linguistics Department, UNICAMP - University of Campinas, \\ Campinas, SP, Brazil. \\ 2- PhD, Assistant Professor, Department of Speech-Language Pathology and Audiology, UNESP - Univ. Estadual Paulista - Marília, SP, Brazil. \\ 3- PhD, Full Professor, Department of Speech-Language Pathology and Audiology, Bauru School of Dentistry; Hospital for Rehabilitation of Craniofacial \\ Anomalies (HRAC), University of São Paulo, Bauru, SP, Brazil. \\ 4- PhD, Associate Professor, Linguistics Department, Institute of Language Studies (IEL), UNICAMP - University of Campinas, Campinas, SP, Brazil. \\ 5- DDS, Dentist, Palatal Prosthesis Department, Hospital for Rehabilitation of Craniofacial Anomalies (HRAC), University of São Paulo, Bauru, SP, Brazil. \\ 6- PhD, Assistant Professor, Department of Speech-Language Pathology and Audiology, Bauru School of Dentistry; Hospital for Rehabilitation of Craniofacial \\ Anomalies (HRAC), University of São Paulo, Bauru, SP, Brazil.
}

Corresponding address: Aveliny Mantovan Lima-Gregio - Rua das Opalas, 26 - Jd. São Domingos - 3471-220 - Americana - SP - BRASIL - Phone: 55 19 3407-4383 - e-mail: avelinylima@gmail.com

Received: October 14, 2009 - Modification: July 14, 2011 - Accepted: August 09, 2011

\section{ABSTRACT}

$T^{\text {he }}$ he use of prosthetic devices for correction of velopharyngeal insufficiency (VPI) is an alternative treatment for patients with conditions that preclude surgery and for those individuals with a hypofunctional velopharynx (HV) with a poor prognosis for the surgical repair of VPI. Understanding the role and measuring the outcome of prosthetic treatment of velopharyngeal dysfunction requires the use of tools that allow for documenting preand post-treatment outcomes. Experimental openings in speech bulbs have been used for simulating VPI in studies documenting changes in aerodynamic, acoustic and kinematics aspects of speech associated with the use of palatal prosthetic devices. The use of nasometry to document changes in speech associated with experimental openings in speech bulbs, however, has not been described in the literature. Objective: This single-subject study investigated nasalance and nasality at the presence of experimental openings drilled through the speech bulb of a patient with HV. Material and Methods: Nasometric recordings of the word "pato" were obtained under 4 velopharyngeal conditions: no-opening (control condition), no speech bulb, speech bulb with a $20 \mathrm{~mm}^{2}$ opening, and speech bulb with 30 $\mathrm{mm}^{2}$ opening. Five speech-language pathologists performed auditory-perceptual ratings while the subject read an oral passage under all conditions. Results: Kruskal-Wallis test showed significant difference among conditions $(p=0.0002)$, with Scheffé post hoc test indicating difference from the no-opening condition. Conclusion: The changes in nasalance observed after drilling holes of known sizes in a speech bulb suggest that nasometry reflect changes in transfer of sound energy related to different sizes of velopharyngeal opening.

Key words: Speech acoustics. Palatal obturators. Cleft palate.

\section{INTRODUCTION}

Tissue insufficiency after primary repair of cleft palate (primary palatoplasty) is one cause of velopharyngeal dysfunction (VPD), resulting in hypernasal speech ${ }^{1}$. Palatal fistula, a well-known residual condition that occasionally occurs after primary palatoplasty, may also result in hypernasal speech ${ }^{5}$. In both conditions, the separation between the oral and nasal cavities needs to be treated physically in order to achieve normal speech. In general, surgical procedures (such as palatal pushback, modified sphincter pharyngoplasty, superior-based pharyngeal flap) are generally 
the treatment of choice to correct VPD following primary palatoplasty ${ }^{23}$. Palatal fistula that remain after primary palatoplasty have also been treated surgically. However, the use of prosthetic treatment still remain an important alternative, particularly for older children and adult patients who present with unsolved problems of velopharyngeal function and/or patients with conditions that preclude surgery ${ }^{1-2,14-15}$

Prosthetic management of VPD generally involves the use of an acrylic appliance to cover fistula through the palate and/or the use of a speech bulb for reducing an opening through the velopharyngeal port. The speech bulb provides a barrier separating the oral and nasal cavities and its success depends on functional movement of the lateral and/or posterior pharyngeal walls which need to embrace the bulb in order for velopharyngeal closure to be achieved ${ }^{23}$. Dentists, speech-language pathologists and plastic surgeons need to work as a team particularly when the speech bulb is the treatment of choice for those individuals who learn to produce speech without eliciting the actions of the velopharynx. The use of speech bulbs can be an important strategy to stimulate increased pharyngeal wall function, optimizing further surgical treatment and supplying dental needs all at the same time. Because the speech bulb must have adequate retention and stability, must feel comfortable to the patient, and must support and elicit residual and/or compensatory velopharyngeal muscle function, the collaboration between the prosthodontist and the speech-language pathologists is essential when prosthetic treatment is the alternative of choice for management of VPD or palatal fistula.

Besides the clinical use of prosthetic or combined behavioral-prosthetic management of VDP to achieve adequate speech, prosthetic appliances have also been used in research studies that aimed to improve our understanding of the effect of velopharyngeal openings on speech. Our knowledge regarding this effect has been raised mostly with information gathered from singlesubject studies that experimentally manipulated the size of velopharyngeal openings drilled into prosthetic devices ${ }^{12,16}$. In most studies in this area it is assumed that velopharyngeal openings of different sizes may affect speech differently, with greater opening thought to have more impact on speech than smaller opening sizes.

Sapienza, et al. ${ }^{16}$ (1996) investigated respiratory, laryngeal, and supralaryngeal functions associated with experimental "fistulas". In this study, the authors described a consistent increase of nasal airflow and decrease in intraoral pressure and vocal intensity as the sizes of the experimental holes increased, with laryngeal and respiratory adjustments most apparent with the $30 \mathrm{~mm}^{2}$ hole size as well as when no obturator was in place. Active physiologic adjustment rather than a passive response to oronasal coupling was therefore observed in this study.

Marino, et al. ${ }^{12}$ (2005) conducted a study that showed physiological adjustments at presence of palatal opening. This study investigated immediate and long-term changes in tongue movement with experimental "fistulas" and revealed that size and location affected tongue movement direction, with larger sizes resulting in greater changes in movement. Particularly, this study have showed that although an immediate response (observed as soon as the experimental "fistula" was created) can be interpreted as indicative of the subject's attempts to move the tongue towards the opening (e.g. to compensate for air loss), the findings following a sustained perturbation (observed after the subject used the obturator with the "fistula" for five days) suggested that with time, other physiological adjustments may help reestablish the requirements to balance air pressure and resonance. Lima-Gregio and colleagues ${ }^{10}(2010)$ investigated frequency

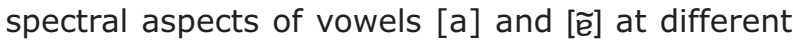
velopharyngeal openings $\left(10 \mathrm{~mm}^{2}, 20 \mathrm{~mm}^{2}\right.$ and 30 $\mathrm{mm}^{2}$ ). Their findings revealed that at the presence of the larger openings $\left(20 \mathrm{~mm}^{2}\right.$ and $\left.30 \mathrm{~mm}^{2}\right)$ the F2 values obtained for the oral vowel [a] were similar to the values obtained for the nasal vowel [ $]$ ], which the authors interpreted as indicative of tongue adjustments (posteriorization).

The above studies described the use of several objective measurements including aerodynamic, acoustic and kinematics to document changes associated to perturbations size introduced during speech while manipulating experimental openings in palatal obturators. Changes in nasalance measures associated to experimental openings, however, have not been described.

The relationship between nasality and nasalance has been the subject of several studies, with data suggesting that many factors can affect these variables. Some authors ${ }^{9}$ reported the possibility of variability due to sources such as equipment, test procedures, between- and within-subjects as well as subject performance. Therefore, while investigating nasalance scores obtained in the presence of prosthesis, one should be aware of possible effects of this device in nasalance results. That is, because nasalance is calculated on the basis of an acoustic ratio of nasal to total oral+nasal acoustic energy, these scores are subject to changes in the impedance in the vocal tract which may affect transmission of acoustic energy $y^{17,19}$.

Scarsellone, et al. ${ }^{17}$ (1999) reported an increase in nasalance score associated to the use of maxillary dentures for 20 women. The authors suggested that the placement of the denture altered oral cavity 
dimensions, affecting acoustical impedance of the oral cavity leading to higher nasalance scores. While statistically significant, clinically the changes in scores were minimal and the authors argued that nasalance changes could be explained by within-subjects variability. Based on their findings, the authors suggested the need for careful use of nasometry for investigated speech resonance, when dealing with patients wearing any obturator (speech bulb or palatal lifts).

Some authors ${ }^{19}$ investigated the relationship between nasalance and palatal lift prosthesis (PLP), which is a prosthesis indicated to elevate the entire velum to a position in the nasopharynx close to speech function, without developing a speech bulb. Findings from this study, however, did not agree with those reported previously ${ }^{17}$. These authors reported that the introduction of the PLP resulted in a consistent and significant decrease in the nasalance scores for all 43 subjects studied. The authors commented that even though the score decreased with the PLP, still a significant difference was found when mean nasalance with PLP was compared with normative data. Also the instrumental findings (indicative of hypernasality) were not consistent with perceptual measures of normal speech nasality (observed by listeners). All participants in Tachimura, Kotani and Wada ${ }^{19}$ (2004) study had repaired cleft palate with residual structural abnormalities (scar tissue) and also had reduced velopharyngeal space with the lift in place, suggesting the possibility of altered transpalatal and transvelum transfer of acoustical energy into nasal cavity. The authors explained that, different than a denture (that only may alter oral impedance), a lift may change oral and also pharyngeal resonance, narrowing the velopharynx, decreasing the size of oral cavity and altering transpalatal and transvelum acoustic transmission.

Further information regarding changes in nasalance associated to the use of palatal prosthesis is still warranted. It is unknown at this moment whether nasometric data can be used for predicting changes in size of velopharyngeal openings. This single-subject study, therefore, investigated nasalance and nasality at the presence of experimental openings drilled through a speech bulb. Since the participant of this study presented normal resonance while wearing her speech bulb prosthesis, we assumed that the introduction of experimental holes of known sizes in the bulb would result in hypernasal resonance and increased nasalance scores. The comparison between the nasometric measures prior to the experimental openings to the measures obtained with the bulb with different sizes of openings allowed us testing the hypothesis that different openings would result in different measures of nasalance.

\section{MATERIAL AND METHODS}

\section{Participant}

The Ethics Committee of the Hospital for Research and Rehabilitation of Craniofacial Anomalies approved this study, which was conducted following the principles outlined in the Declaration of Helsinki (Ethics Committee Approval Number: 038/2004). A 17-year-old female with a cleft of the hard and soft palate served as the single subject in this investigation. The patient remained with velopharyngeal insufficiency (VPI) after primary correction of the cleft palate and was fitted with a speech bulb to optimize velopharyngeal functioning during a speech bulb reduction program, since the prognosis for successful surgical repair was poor due to limited movement at the velopharynx.

At the time of this experiment, the participant had been successfully wearing a removable speech bulb for over 6 months and her speech (voice, articulation and resonance) was judged to be normal by herself as well as by 5 speech-language pathologists (SLPs), family and peers. The patient presented no laryngeal, respiratory, otologic, audiologic or speech conditions during the study and was free of allergies, colds and did not use nasal decongestants while the study was conducted.

\section{Replica of the speech bulb}

To create controlled openings through the speech bulb, a replica of the subject's original prosthesis with a speech bulb was obtained as described previously ${ }^{12,16}$. The subject was instructed to wear the replica during one week before initiating data collection to adapt to any uncontrollable variances between the replica and original prosthesis. The subject herself expressed that her speech was not different while wearing the replica prosthesis. Additionally, nasendoscopic evaluation was conducted by an experienced SLP while the subject produced short phrases with pressure consonants, showing that the replica bulb was well fitted and adapted, fostering velopharyngeal closure during adequate speech production.

\section{Control and experimental conditions}

Speech samples were obtained under one control and three experimental conditions. The control condition included data recorded while the subject was wearing the replica with noopening (NO) drilled through it, resulting in normal velopharyngeal function during speech. The three experimental conditions included: (1) no prosthesis (NP), (2) replica with a $20 \mathrm{~mm}^{2}$ hole $\left(20 \mathrm{~mm}^{2}\right)$, and (3) replica with a $30 \mathrm{~mm}^{2}$ hole $\left(30 \mathrm{~mm}^{2}\right.$ ). The holes were drilled through the experimental speech bulbs (Figure 1) using a standard shop drill press with a bit area size approximating $20 \mathrm{~mm}^{2}$ 
and $30 \mathrm{~mm}^{2}$. This hole sizes were selected based on earlier research indicating that a hole as small as $20 \mathrm{~mm}^{2}$ resulted in a substantial changes to speech $^{12,16}$. In the present study, speech recordings for all conditions (control and experimental) were obtained at the same session, in random order.

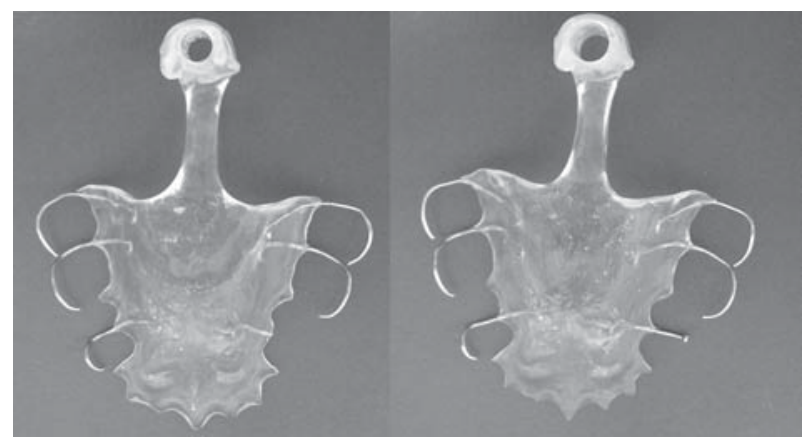

Figure 1- Prostheses with $20 \mathrm{~mm}^{2}$ (left) and $30 \mathrm{~mm}^{2}$ (right) holes drilled through the speech bulb

\section{Nasometric assessment}

The Brazilian Portuguese word "pato" (meaning: duck) repeated within the carrier phrase "Digo bojudo" ("Say stuffed ") was used as stimuli for nasometric assessment in this study. Although nasometry is usually conducted with longer passages, data on shorter segments has been already reported for Portuguese speakers ${ }^{4}$. Additionally, the use of words and short phrases with controlled phonetic homogeneity for nasometric assessment is frequently used at Hospital for Rehabilitation of Craniofacial Anomalies (HRAC) 4 and also proposed with the Snap Test ${ }^{8}$.

A SLP, experienced with nasometry, obtained nasalance scores for the participant using Kay Elemetrics' Nasometer (Model 6220-2, 1994)7. Calibration, data recording and calculation of nasalance scores were done according to the procedures described in the Nasometer's Manual, with an adaptation of the positioning of the of the Nasometric sound separator plate. The participant practiced the production of the phrase after instruction from the examiner and repeated the stimuli six times for each of the 4, leading to 24 samples. Another examiner assured stable positioning of the Nasometer's separator plate between the upper lip and nostril of the speaker. The 6 repetitions recorded were used for calculating a mean nasalance for the word "pato". The SLP who conducted the nasometric assessment was blinded to the different appliance conditions.

\section{Perceptual ratings of speech nasality}

For ratings of speech nasality a standardized oral passage in Brazilian Portuguese ${ }^{18}$ was used, facilitating SLPs speech ratings of the presence or absence of hypernasality. The text was presented in writing and the participant read it once under each of the conditions studied. Five SLPs with at least two years of experience in rating speech nasality across an array of patients with resonance disorders related to VPD assessed speech nasality. The SLPs independently performed a live auditory-perceptual evaluation of nasality while the subject read the oral passage 4 times (one for each condition) at the same day the nasalance measures and nasoendoscopic assessment of velopharynx were taken. Listeners were not aware of the bulb condition during ratings. They were asked to indicate presence or absence of hypernasality in a scoring sheet without regard to the presence of other identifiable differences such as inappropriate nasal air emission, weak pressure consonants, or changes in pitch, loudness, duration and prosody.

\section{Data analysis}

Mean nasalance, standard deviation, minimum, and maximum were obtained for the word "pato" under controls and experimental conditions. Kruskal-Wallis statistical test was used to evaluate the effect of conditions on nasometric measures. Statistical significance was set at $p<0.05$. Scheffé post hoc test was used for post hoc testing. Percentage agreement between majority of five listeners during auditory-perceptual ratings of nasality were calculated for each condition tested.

\section{RESULTS}

A summary of the findings of the present study for all conditions (NO, $20 \mathrm{~mm}^{2}, 30 \mathrm{~mm}^{3}$, NP) has been presented in Table 1 including nasalance mean and standard deviation (SD), nasalance minimum and maximum values, auditory-perceptual rating of nasality (normal or hypernasal) and listener's agreement during perceptual ratings.

Particularly for this study, the mean nasalance obtained with the speech bulb with no openings (NO) was lower than the mean obtained with openings in the bulb $\left(20 \mathrm{~mm}^{2}, 30 \mathrm{~mm}^{2}\right)$ and without prosthesis (NP). While the mean nasalance with 20 $\mathrm{mm}^{2}$ opening was lower than the mean with the 30 $\mathrm{mm}^{2}$ opening, mean nasalance without prosthesis (NP) did not follow this pattern of greater nasalance with greater opening.

Normal speech nasality was identified by all 5 SLPs when the participant read the standardized passage wearing the bulb with no opening (NO). Hypernasal speech was identified by all SLPs for the $30 \mathrm{~mm}^{2}$ and NP conditions and by the majority of the SLPs $(60 \%)$ for the $20 \mathrm{~mm}^{2}$. Nasometric findings agreed with listeners ratings when hypernasality was present. For the NO condition however, the instrument indicated hypernasality while listeners 
Table 1- Summary of findings of present study with conditions, mean nasalance scores and Standard Deviation (SD), minimum and maximum values of nasalance, perceptual rating of nasality and listener's agreement during perceptual ratings

\begin{tabular}{ccccc}
\hline Condition & Mean \pm SD & Min-Max & Speech & $\%$ agreement \\
\hline NO & $44 \pm 9$ & $36-58$ & Normal & $100 \%$ \\
$20 \mathrm{~mm}^{2}$ & $55 \pm 2$ & $52-58$ & Hypernasal & $60 \%$ \\
$30 \mathrm{~mm}^{2}$ & $56 \pm 2$ & $54-60$ & Hypernasal & $100 \%$ \\
NP & $53 \pm 5$ & $48-60$ & Hypernasal & $100 \%$ \\
\hline
\end{tabular}

Table 2- Summary of findings of present and previous studies for the conditions with and without prosthesis, including number of samples, speech stimuli, nasalance scores (mean, Standard Deviation)

\begin{tabular}{|c|c|c|c|}
\hline Language and conditions studied & $\mathbf{N}$ & Stimuli & Mean $\pm S D$ \\
\hline Portuguese norms ${ }^{4}$ & 30 & Single Word & $25 \% \pm 10 \%$ \\
\hline With prosthesis NOpresent study & 6 & Single Word & $44 \% \pm 9 \%$ \\
\hline Without prosthesis NPresent study & 6 & Single Word & $53 \% \pm 5 \%$ \\
\hline Japanese norms ${ }^{19}$ & 100 & Oral Phrases & $9 \% \pm 4 \%$ \\
\hline With palatal lift 20 & 43 & Oral Phrases & $17 \% \pm 8 \%$ \\
\hline Without palatal lift²0 & 43 & Oral Phrases & $34 \% \pm 13 \%$ \\
\hline English norms ${ }^{7}$ & 117 & Oral Phrases & $16 \% \pm 5 \%$ \\
\hline With dentures ${ }^{17}$ & 20 & Oral Phrases & $12 \% \pm 5 \%$ \\
\hline Without dentures ${ }^{17}$ & 20 & Oral Phrases & $11 \% \pm 5 \%$ \\
\hline
\end{tabular}

perceived normal speech nasality.

Kruskal-Wallis statistical test was used to evaluate the effect of conditions on nasometric measures revealing significant difference between conditions $(p=0.0002)$. Scheffé post hoc test indicated that the difference at the $5 \%$ significance level was between the no opening condition and all 3 experimental conditions.

\section{DISCUSSION}

This study investigated nasalance and nasality at the presence of different experimental openings of controlled sizes drilled through a speech bulb. The findings of this study were compared to nasalance norms for Portuguese language ${ }^{4}$ and to previous studies in Japanese and English languages that investigated nasalance scores at the presence of prosthesis ${ }^{17,19}$. Table 2 presents a summary of nasalance scores for all studies involving palatal prosthesis along with nasalance norms in the languages studied.

Even at the presence of normal resonance as indicated by listeners, the mean nasalance obtained for this study when the participant had the prosthesis with no-openings (44\%) was much higher than normative data reported for Portuguese language ${ }^{4}$. DiNinno, et al. ${ }^{4}$ (2001) reported mean nasalance during word production of $25 \%$ (SD: $10 \%$ ) for adult females and 21\% (SD: $8 \%$ ) for adult males. The present study's mean was also higher than the proposed cut-off value of $27 \%$ used for oral samples for Portuguese language ${ }^{21}$. This result suggests that even at the presence of speech rated as normal while the participant was wearing a replica of her speech bulb (with no openings) a lack of complete velopharyngeal closure may actually still exist, leading to nasal air emission which was not identified by the listeners but may have been pick-up by the nasometer. Nasal air emission, therefore, may be one explanation for higher nasalance scores found with the control condition while compared to norms.

While mean nasalance found for the participant with prosthesis with no opening (NO) was indicative of presence of hypernasality, listeners agreed $100 \%$ that nasality was normal. This inconsistency between instrumental and perceptual findings may be partly explained by the use of different stimuli during evaluation, since nasalance was obtained for a single word produced within a short carrier phrase and nasality was rated during reading of an oral passage. Tachimura, Kotani and Wada ${ }^{19}$ (2004) also reported scores significantly above norms for a group with the PLP, while investigating the relationship between nasalance and the use of a palatal lift. While the introduction of the lift resulted in a significant decrease in the nasalance scores for all subjects, the authors indicated that the scores with the lift still were significantly greater than the norms disagreeing with their listeners' perceptual findings of normal nasality. Instrumental and 
perceptual assessments in the study of Tachimura, Kotani and Wada ${ }^{19}$ (2004) also used different speech stimuli, with nasality rated during conversational speech and nasalance obtained during production of a short passage. While the authors used a palatal lift for correction of VPI, the present study used a speech bulb (obturator), leading to the possibility of differences in the transfer of energy and oral/ pharyngeal impedance between both studies.

Considering the relationship between nasalance and changes of impedance resulting from the introduction of the prosthesis in oral cavity and velopharynx, the findings of increased nasalance with the speech bulb with no openings (when compared to norms) obtained in our study agreed with those reported in the literature ${ }^{17,19}$. While Scarsellone, Rochet and Wolfaardt ${ }^{17}$ (1999) described a significant increase in nasalance scores associated to the use of maxillary dentures, the difference between the condition with dentures $(12 \%)$ and without dentures $(11 \%)$ was only $1 \%$ and the mean scores at both condition were still under the norms published for English speakers $(16 \%)^{7}$. Tachimura, Kotani and Wada ${ }^{19}(2004)$ study stated that mean nasalance scores without the lift was higher $(34 \%)$ than the scores with the lift in place $(17 \%)$ for a group of patients who had their velopharyngeal inadequacy treated with palatal lifts. These authors ${ }^{19}$, however, noted that the mean nasalance with the lift in place (17\%), was a little higher than the nasalance norms for the Japanese language $^{20}(9 \%)$, speculating that an increase in speech nasalance values could be the result of changes in transpalatal and transvelum transfer of acoustic energy. Therefore, higher nasometric values obtained in our study could be the result, at least in part, of changes in transpalatal and transvelum transfer of acoustic energy due to the presence of acrylic material used in the prosthetic appliance. Future studies, however, are still needed to investigate the effect of material and shape prosthetic devices in nasalance scores.

Many aspects of speech may change/adjust when perturbations are introduced to the speech system during sound production ${ }^{3}$. Nasometry is limited to documenting only changes in the transfer of acoustical energy. If we interpret the immediate changes in nasalance observed after drilling holes of known sizes as representative of linear responses to changes in size of the velopharyngeal space, we can consider nasalance to be sensitive to changes in velopharyngeal function related to different types of physical management (prosthetic and surgical). But while change in mean nasalance observed while controlling the size of the openings was linear, it showed only a $1 \%$ increase in nasalance score for the $30 \mathrm{~mm}^{2}$ opening condition (56\%) when compared to the $20 \mathrm{~mm}^{2}$ opening condition (55\%).
This change in score among these 2 conditions was smaller than the SDs reported in this study agreeing with perceptual assessment of speech which revealed hypernasality for both conditions. This calls for careful interpretation of these findings. The mean nasalance obtained when no speech bulb was in place (53\%) was lower than the means found for the 2 conditions with experimental opening (55\% and $56 \%$ ), which can be interpreted as suggestive of the possibility of active physiologic adjustments, such as reported by ${ }^{10,12,16}$. As the later authors described respiratory, laryngeal, and supralaryngeal physiological adjustments may take place as the conditions within the speech system changes. Future studies monitoring presence of adjustments such as increase or decrease in respiratory support, elevation or depression of larynx, adjustments in tongue posture or mouth opening can further our understanding of the strategies used by the speakers when perturbations are introduced to the speech system during sound production.

While perceptually and instrumentally monitoring the outcome of physical treatment of VPD is needed for efficacy based practice ${ }^{11,13}$ the use of nasometry to document outcome has to be done carefully. The nonlinearity of findings reported in this study may be associated to the fact that the Nasometer captures two types of acoustic energy - one associated with sound resonance and the other associated with noise resulting from nasal emission ${ }^{6,22}$, and nasal air emission may not always be audible to listeners but may be detected by the instrument. The present findings point to the need for future studies while controlling several variables including: the use of equal and longer speech stimuli, the use of speech bulbs made of different material/configuration/shapes, the use of other measures of transpalatal and transvelum transfer of acoustical energy, and the use of measures to monitor physiologic adjustments.

\section{CONCLUSION}

The changes in nasalance observed after drilling holes of known sizes in a speech bulb suggest that nasometry reflect changes in transfer of sound energy related to different sizes of velopharyngeal opening. 


\section{REFERENCES}

1- Bzoch KR, ed. Communicative disorders: related to cleft lip and palate. $5^{\text {th }}$ ed. Austin: Pro-Ed; 2004.

2- Carvalho-Teles V, Pegoraro-Krook MI, Lauris JRP. Speech evaluation with and without palatal obturator in patients submitted to maxillectomy. J Appl Oral Sci. 2006;14(6):421-6.

3- Dalston RM. The use of nasometry in the assessment and remediation of velopharyngeal inadequacy. In: Bzoch $K R$, ed. Communicative disorders related to cleft lip and palate. $5^{\text {th }}$ ed. Austin: Pro-Ed; 2004. p. 495-516.

4- Di Ninno CQMS, Vieira JM, Teles-Magalhães LC, Padovani CR, Pegoraro-Krook MI. Nasalance values identification in normal Brazilian Portuguese speakers. Pró-Fono. 2001;13(1):71-7.

5- Henningsson G, Isberg A. Influence of palatal fistulae on speech and resonance. Folia Phoniatr (Basel). 1987;39:183-91.

6- Karnell MP. Nasometric discrimination of hypernasality and turbulent nasal airflow. Cleft Palate Craniofac J. 1995;32(2):146-8. 7- Kay Elemetrics Corporation. Instruction manual for the nasometer model 6200. Lincoln Park: Kay Elemetrics Corporation; 1994.

8- Kummer AW. Velopharyngeal dysfunction (VPD) and resonance disorders. In: Kummer AW. Cleft palate and craniofacial anomalies: effects on speech and resonance. San Diego: Singular Learning; 2001.

9- Lewis KE, Watterson T, Blanton A. Comparison of short-term and long-term variability in nasalance scores. Cleft Palate Craniofac J. 2008;45(5):495-500.

10- Lima-Gregio AM, Dutka-Souza JCR, Marino VCC, Pegoraro-

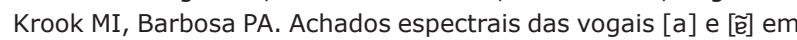
diferentes aberturas velofaríngeas. Pró-Fono. 2010;22(4):515-20. 11- Lipira A, Grames LM, Molter D, Govier D, Kane A, Woo A. Videofluoroscopic and nasendoscopic correlates of speech in velopharyngeal dysfunction. Cleft Palate Craniofac J. 2011;48(5):550-60.

12- Marino VCC, Williams WN, Wharton PW, Paulk MF, DutkaSouza JC, Schulz GM. Immediate and sustained changes in tongue movement with an experimental palatal "fistula": a case study. Cleft Palate Craniofac J. 2005;42(3):286-96.
13- Pegoraro-Krook MI, Dutka-Souza JC, Marino VC. Nasoendoscopy of velopharynx before and during diagnostic therapy. J Appl Oral Sci. $2008 ; 16(3): 181-8$

14- Pinto JH, Dalben GS, Pegoraro-Krook MI. Speech intelligibility of patients with cleft lip and palate after placement of speech prosthesis. Cleft Palate Craniofac J. 2007;44(6):635-41.

15- Pinto JH, Pegoraro-Krook MI. Evaluation of palatal prosthesis for the treatment of velopharyngeal dysfunction. J Appl Oral Sci. 2003;11(3):192-7.

16- Sapienza CM, Brown WS, Williams WN, Wharton PW, Turner GE. Respiratory and laryngeal function associated with experimental coupling of oral and nasal cavities. Cleft Palate Craniofac J. 1996;33(2):118-26.

17- Scarsellone JM, Rochet AP, Wolfaardt JF. The influence of dentures on nasalance values in speech. Cleft Palate Craniofac J. $1999 ; 36(1): 51-6$.

18- Suguimoto MLCP, Pegoraro-Krook MI. Nasalance values of normal adults, Brazilian Portuguese speakers. Pró-Fono. 1996;8(1):7-12.

19- Tachimura T, Kotani Y, Wada T. Nasalance scores in wearers of a palatal lift prosthesis in comparison with normative data for Japanese. Cleft Palate Craniofac J. 2004;41(3):315-9.

20- Tachimura T, Mori C, Hirata S, Wada T. Nasalance score variation in normal adult Japanese speakers of Mid-West Japanese dialect. Cleft Palate Craniofac J. 2000;37(5):463-7.

21- Trindade IEK, Genaro KF, Dalston RM. Nasalance scores of normal Brazilian Portuguese speakers. Braz J Dysmorphol Speech Hear Disord. 1997;1(1):23-34.

22- Watterson T, Lewis KE, Deutsch C. Nasalance and nasality in low pressure and high pressure speech. Cleft Palate Craniofac J. $1998 ; 35(4): 293-7$.

23- Williams WN, Henningsson G, Pegoraro-Krook MI. Radiographic assessment of velopharyngeal function for speech. In: Bzoch KR, ed. Communicative disorders related to cleft lip and palate. $5^{\text {th }}$ ed. Austin: Pro-Ed; 2004. 